\title{
Uso do WhatsApp@ por gestores de serviços de saúde
}

Use of WhatsApp ${ }^{\circledR}$ by health care managers

Uso del WhatsApp® por administradores de servicios de salud

Renata Olszewski Savio ${ }^{1}$ (i) https://orcid.org/0000-0001-6746-5345 Maynara Fernanda Carvalho Barreto ${ }^{1}$ @ https://orcid.org/0000-0002-3562-8477

Danielli Rafaeli Candido Pedro ${ }^{1}$ io https://orcid.org/0000-0003-4141-1220

Raquel Gvozd Costa ${ }^{1}$ id https://orcid.org/0000-0002-5816-8215

Mariana Angela Rossaneis ${ }^{1}$ ib https://orcid.org/0000-0002-8607-0020

Larissa Gutierrez de Carvalho Silva ${ }^{1}$ in https://orcid.org/0000-0003-0209-930X

Patrícia Aroni' ${ }^{1}$ if https://orcid.org/0000-0001-5092-2714

Maria do Carmo Fernandez Lourenço Haddad ${ }^{1}$ io https://orcid.org//0000-0001-7564-8563

Como citar:

Savio RO, Barreto MF, Pedro DR, Costa $R G$, Rossaneis $M A$, Silva $L G$, et al. Uso do WhatsApp $®$ por gestores de serviços de saúde. Acta Paul Enferm. 2021;34:AAPE001695.

DOI

http://dx.doi.org/10.37689/actaape/2021A0001695

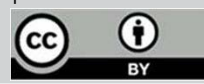

Descritores

Comunicação; Disseminação de informação; Administração de serviços de saúde; Gestor de

saúde; Rede socia

Keywords

Communication; Information dissemination; Health services administration; Health manager; Social networking

Descriptores

Comunicación; Difusión de la información Administración de los servicios de salud; Gestor de

salud; Red social

Submetido

1 de Julho de 2020

Aceito

9 de Dezembro de 2020

Autor correspondente

Renata Olszewski Savio

E-mail: renatawski@gmail.com

\section{Resumo}

Objetivo: Descrever como gestores de serviços de diferentes níveis de atenção à saúde utilizam 0 aplicativo WhatsApp $®$ na sua atuação profissional.

Métodos: Estudo qualitativo, do tipo descritivo, desenvolvido por entrevistas semi-estruturadas, realizadas com 14 profissionais gestores de serviços de saúde, de um município localizado no Norte do estado do Paraná, Brasil. Para análise dos dados foi utilizado o software IRAMUTEQ, pelo método de Classificação Hierárquica Descendente. Os componentes principais de cada classe foram analisados a partir de um referencial teórico de comunicação.

Resultados: A partir das respostas dos participantes, o corpus textual foi dividido em seis classes, respectivamente: a desconfiança do emissor sobre 0 uso da informação enviada; 0 impacto da agilidade e resolutividade na jornada de trabalho dos gestores; 0 uso do WhatsApp $®$ como estratégia de integração dos serviços e profissionais envolvidos na gestão do serviço; a utilidade do WhatsApp $®$ como ferramenta gerencial; as principais potencialidades e fragilidades no uso do WhatsApp $®$ e a percepção da utilidade do aplicativo em decisões que influenciam o processo de trabalho.

Conclusão: 0 estudo descreveu como os gestores de serviços de saúde utilizam o WhatsApp® na sua atuação profissional, evidenciando a importância do aplicativo enquanto ferramenta estratégica na gestão dos serviços de saúde, a partir do compartilhamento e integração de informações em tempo real.

\section{Abstract}

Objective: To describe how service managers of different levels of health care use the WhatsApp ${ }^{\circledR}$ application in their professional practice.

Methods: Qualitative, descriptive study developed by means of semi-structured interviews conducted with 14 health care management professionals from a municipality located in the north of the state of Paraná, Brazil. The IRAMUTEQ software was used for data analysis through the Descending Hierarchical Classification method. The main components of each class were analyzed based on a theoretical framework of communication.

Results: Based on participants' responses, the textual corpus was divided into the following six classes: the sender's mistrust in the use of the information sent; the impact of agility and resoluteness on managers' working hours; the use of WhatsApp ${ }^{\circledR}$ as a strategy for integrating services and professionals involved in service management; the utility of WhatsApp ${ }^{\circledR}$ as a management tool; the main strengths and weaknesses in the use of WhatsApp ${ }^{\circledR}$; and perception of the application utility in decisions that influence the work process.

Conclusion: The study described how health care managers use WhatsApp ${ }^{\circledR}$ in their professional practice, highlighting the importance of the application as a strategic tool in health care management, from real-time sharing and integration of information.

Universidade Estadual de Londrina, Londrina, PR, Brasil.

Conflitos de interesse: nada a declarar. 


\section{Resumen}

Objetivo: Describir de qué forma los administradores de servicios de diferentes niveles de atención en salud utilizan la aplicación WhatsApp $®$ en su trabajo profesional.

Métodos: Estudio cualitativo, tipo descriptivo, llevado a cabo mediante entrevistas semiestructuradas, realizadas a 14 profesionales administradores de servicios de salud de un municipio ubicado al norte del estado de Paraná, Brasil. Para el análisis de datos se utilizó el software IRAMUTEQ, con el método de clasificación jerárquica descendiente. Los componentes principales de cada clase fueron analizados a partir de un marco referencial teórico de comunicación.

Resultados: A partir de las respuestas de los participantes, se dividió el corpus textual en seis clases, a saber: la desconfianza del emisor sobre el uso de la información enviada; el impacto de la agilidad y resolución en la jornada de trabajo de los administradores; el uso del WhatsApp® como estrategia de integración de los servicios y profesionales involucrados en la gestión del servicio; la utilidad del WhatsApp $®$ como herramienta administrativa; las principales posibilidades y fragilidades del uso de WhatsApp $®$, y la percepción de la utilidad de la aplicación en decisiones que influyen en el proceso de trabajo.

Conclusión: El estudio describió de qué forma los administradores de servicios de salud usan el WhatsApp® en su trabajo profesional y demostró la importancia de la aplicación como herramienta estratégica para la gestión de servicios de salud, a partir de la posibilidad de compartir e integrar información en tiempo real.

\section{Introdução}

As mídias sociais correspondem a canais de informação e comunicação, que possibilitam a interaçáo entre usuários por meio de compartilhamento de informações, criação de conteúdo, expressão de opiniôes e participação em discussões, por exemplo. ${ }^{(1)}$ As redes sociais são uma das categorias mais conhecidas e utilizadas, pois permitem conectar indivíduos por meio de plataformas online. ${ }^{(2)}$

$\mathrm{O}$ que transformou a rotina de muitas pessoas, não só na esfera pessoal, como nas relaçóes profissionais, por favorecer uma comunicação instantânea, que permite uma maior aproximação entre os participantes, tornando possível novos métodos de trabalho, com custo-efetividade satisfatório. ${ }^{(3)}$

Compete ao gestor as funçóes de planejamento, organização, direção e controle, considerando que suas atitudes e decisóes influenciam diretamente nas atividades e resultados obtidos nas instituiçóes. ${ }^{(4)}$ Ferramentas como o WhatsApp ${ }^{\oplus}$, auxiliam esses profissionais em cargos e funçóes de gestão a manter uma comunicação efetiva com os demais envolvidos no processo de trabalho, contribuindo no desenvolvimento da organização, principalmente nas atividades do cotidiano, além de determinar a disseminação de informaçóes pertinentes às açóes da gestão aos demais colaboradores. ${ }^{(5)}$

De acordo com o site oficial da empresa WhatsApp ${ }^{\oplus}$, o aplicativo é gratuito e dispóe de suporte para o envio e recebimento de arquivos de mídia, como fotos, vídeos, documentos, compartilhamento de localização, textos e chamadas de voz, de forma simples e segura, utilizado por mais de 1 bilhão de pessoas, em mais de 180 países ao redor do mundo. ${ }^{(6)}$

$\mathrm{Na}$ área da saúde, se popularizou o uso de tecnologias de comunicação, tornando dinâmico o fluxo de dados e informaçóes relacionados a assuntos gerenciais, tomadas de decisão e comunicação entre equipes e gestores, além de contribuir para o rápido acesso a notícias e fatos importantes referentes ao trabalho, o que possibilita respostas e intervençóes em tempo hábil. ${ }^{(7)}$

Sabendo da funcionalidade do aplicativo para diversas finalidades, estudos foram realizados com o objetivo de identificar a utilização das redes sociais na educação em saúde, ${ }^{(8,9)}$ assim como seu uso nos processos de trabalho em enfermagem ${ }^{(2)}$ e entre profissionais da saúde, ${ }^{(10)}$ no entanto, não foram encontrados estudos referentes ao uso de redes sociais, sobretudo o uso de WhatsApp ${ }^{\oplus}$, por gestores de serviços de saúde.

Considerando a relevância de analisar como e por quais motivos o gestor de saúde utiliza as ferramentas tecnológicas como o WhatsApp ${ }^{\oplus}$ em seu processo de trabalho, este estudo tem por objetivo descrever como gestores de serviços de diferentes níveis de atenção à saúde utilizam o aplicativo WhatsApp ${ }^{\circledast}$ na sua atuação profissional.

\section{Métodos}

Trata-se de estudo qualitativo, do tipo descritivo, realizado em diferentes serviços de saúde de um município localizado no Norte do estado do Paraná, Brasil. Utilizou-se como ferramenta norteadora para escrita 
desse artigo o Consolidated criteria for reporting qualitative research (COREQ), contendo 32 itens. $^{(11)}$

A população desse estudo foi constituída intencionalmente, a fim de que tivesse gestores que atuam nos três níveis de atenção à saúde de serviços de saúde público e filantrópico. Os participantes foram selecionados a partir de informaçóes coletadas em sites institucionais, disponíveis de domínio público, com o cuidado de não expor as instituições onde o profissional trabalha.

A coleta de dados aconteceu entre maio e junho de 2019. Os participantes selecionados foram convidados pessoalmente pela pesquisadora principal, que inicialmente identificou-se e apresentou os objetivos da pesquisa. Não houve recusas aos convites. A entrevista foi agendada no local de trabalho de cada gestor, conforme escolha dos mesmos, respeitando-se o horário de trabalho, gravada em dispositivo de áudio, na presença somente do pesquisador e gestor, garantindo sigilo na realização da entrevista. A duração das entrevistas variou entre três à 14 minutos. Posteriormente, o áudio foi transcrito e, após a transcrição, excluído permanentemente do dispositivo de áudio utilizado.

Fizeram parte do estudo, 14 gestores. Destes, cinco atuavam em hospitais públicos de nível secundário, quatro em hospital público de nível terciário, um em um hospital filantrópico de nível terciário e quatro em serviços públicos vinculados a secretaria de saúde do município em estudo. Os participantes preencheram um formulário para caracterização sociodemográfica e laboral que incluiu idade, sexo, tempo de formação profissional, nível de especialização, tipo de vínculo empregatício, tempo de atuação na instituição, cargo de gestão que ocupa, jornada de trabalho, entre outros.

Para responder ao objetivo da pesquisa, foram utilizadas as seguintes perguntas: 1- Como você utiliza o WhatsApp ${ }^{\circledast}$ na sua atuação profissional? 2- Você considera o uso do aplicativo essencial em sua atuação profissional? Por que? 3- Você já tomou ou costuma tomar decisóes importantes via WhatsApp ${ }^{\oplus}$ ? 4- Qual sua percepção sobre a segurança e privacidade do aplicativo? 5- Quais as potencialidades e as fragilidades no uso do WhatsApp ${ }^{\oplus}$ no seu processo de trabalho?
Foram entrevistados 14 gestores, após a repetição contínua dos dados que responderam aos objetivos do estudo, garantido a representatividade igualitária entre gestores dos serviços de saúde dos três níveis de atenção.

Os dados passaram por análise de conteúdo, com apoio do Software IRAMUTEQ ${ }^{(12)}$ (Interface de R pour les Analyses Multidimensionnelles de Textes et de Questionnaires), por meio do método de Classificação Hierárquica Descendente (CHD), o corpus textual foi dividido em seis classes, a partir da estruturação dos segmentos de texto ou unidades de contexto elementar (UCE), classificados de acordo com os vocabulários de maior frequência e o grau de associaçáo com cada classe, por meio do teste qui-quadrado. ${ }^{(13)}$

Os componentes principais de cada classe foram analisados a partir do referencial teórico, que corresponde ao capítulo "Comunicação" do livro "Comportamento Organizacional" de Stephen P. Robbins. ${ }^{(14)}$

O estudo foi aprovado pelo Comitê de Ética em Pesquisa Envolvendo Seres Humanos (CEP) de uma universidade localizada no Norte do estado do Paraná, Brasil, que atende a Resolução 466/2012 e Resolução Complementar 510/2016 do Conselho Nacional de Saúde do Ministério da Saúde, em defesa dos sujeitos participantes de pesquisa, sob o Parecer $n^{\circ} 3.251 .487$. Os participantes selecionados foram esclarecidos quanto à pesquisa e assinaram o Termo de Consentimento Livre e Esclarecido.

Os entrevistados foram indicados pela letra $G$, de gestor(a), seguido pelo número correspondente de acordo com a ordem cronológica de participação (G01, G02, G03...), o que garante o anonimato e confidencialidade das informaçôes obtidas durante as entrevistas.

\section{Resultados}

Em relação à caracterização sociodemográfica dos gestores, dentre os 14 participantes, nove eram do sexo feminino e cinco do sexo masculino, com idade entre 31 e 71 anos. No que se refere à graduação, três gestores tiveram sua formação profissional em 
administração, nove em enfermagem, um em medicina e um em secretariado executivo, com o tempo de formaçáo variando entre 10 e 47 anos. Quanto à qualificação dos gestores entrevistados, todos possuíam cursos de pós-graduação lato e stricto sensu.

O tempo de trabalho no cargo de gestor variou entre dois e 35 anos, e no que tange aos cargos ocupados atualmente, correspondem ao cargo de administrador, assessor, coordenador, diretor e superintendente da instituição. Todos os 14 gestores referiram trabalhar em tempo integral, de 40 a 60 horas semanais em média, com renda mensal maior que quatro salários mínimos. Acerca do tipo de vínculo empregatício, um gestor possuía cargo comissionado, 12 eram estatutários e um, pessoa jurídica.

Por meio do método de CHD para análise do conteúdo das entrevistas, o corpus textual foi dividido em dois sub-corpus, que se dividem em outras duas repartiçóes cada. De um dos sub-corpus, obteve-se a Classe 2 (o impacto da agilidade e resolutividade na jornada de trabalho dos gestores), que compóe $20 \%$ do total das UCE e que origina a Classe 4 (utilidade do WhatsApp ${ }^{\oplus}$ como ferramenta gerencial), com 16,4\% das UCE e a Classe 5 (principais potencialidades e fragilidades no uso do WhatsApp ${ }^{\oplus}$ ), que concentra $18,2 \%$.

Do outro sub-corpus, derivou-se a Classe 3 (o uso do WhatsApp ${ }^{\oplus}$ como estratégia de integração dos serviços e profissionais envolvidos na gestão do serviço), que compreende $12,7 \%$ das UCE, e que origina a Classe 1 (a desconfiança do emissor sobre o uso da informação enviada) e 6 (percepção da utilidade do aplicativo em decisóes que influenciam o processo de trabalho), que correspondem a $20 \%$ e $12,7 \%$ das UCE do corpus total respectivamente, conforme o dendograma demonstrado na figura 1 .

A Classe 1, desconfiança do emissor sobre o uso da informaçáo enviada, revelou a insegurança do gestor sobre o uso que o receptor pode fazer da mensagem enviada, e demonstra que o e-mail ainda é considerado o canal formal por excelência entre a maioria dos participantes.

Tem que ter bastante critério com o que a gente escreve porque essa mensagem pode ser encaminhada e interpretada de outra forma. G14

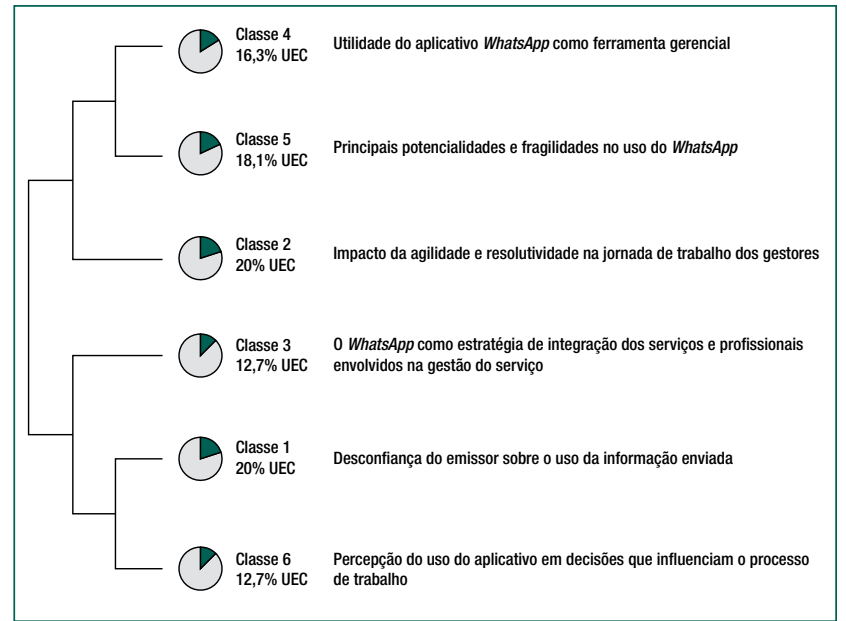

Figura 1. Dendograma das classes

Eu sei que quando você quer oficializar uma coisa tem que ser no mínimo por e-mail porque o Whats $A p p^{\oplus}$ não tem ainda o meio oficial de você tratar as coisas, apesar de que na prática acaba acontecendo. G10

A Classe 2, denominada como o impacto da agilidade e resolutividade na jornada de trabalho dos gestores, evidenciou a importância da comunicação em tempo real na resoluçáo de problemas, que, no entanto, gerou uma cobrança de disponibilidade do gestor fora do horário de trabalho.

Ele tem se tornado uma ferramenta de trabalho, felizmente porque você consegue resolver muito rapidamente muitas coisas, (...) infelizmente por conta dessa velocidade eles cobram que você tenha disponibilidade 24 horas, quando alguém manda uma mensagem, já quer que você responda na hora. G07

Eu uso muito e acho muito positivo o uso. Por exemplo, eu estou falando com você, mas eu posso estar dando uma ordem pra um funcionário lá da manutenção. (...) hoje eu não consigo imaginar a gestão sem o WhatsApp ${ }^{\oplus}$. Mas assim, o imediatismo do Whats $A p p^{\oplus}$ é prejudicial, porque quando eu leio, eu tenho que pensar pra responder, parece que tudo virou urgente. G05

A Classe 3, o WhatsApp ${ }^{\varpi}$ como estratégia de integração dos serviços e profissionais envolvidos na ges- 
tão do serviço, demonstrou que o aplicativo se tornou uma ferramenta fundamental na aproximação das pessoas envolvidas no processo de trabalho, facilitando a atuação do gestor dentro da organização.

$O$ Whats $A p p^{\oplus}$ é uma ferramenta essencial porque ele aproxima muito as áreas dentro do organograma administrativo e extra-muros. G03

Hoje em dia pra você fazer gestão você tem que se comunicar com vários segmentos, com várias pessoas e setores, e o Whats $A p p^{\circledast}$ permite que você faça essa comunicação mais ágil, por ser um elemento dentro de um smartfone, portátil, onde você estiver, você responde, então isso ajuda na facilidade de comunicação e integração, então você fica mais conectado com os setores que você mais precisa ter atuação. G13

A Classe 4, utilidade do WhatsApp ${ }^{\varpi}$ como ferramenta gerencial, se refere ao uso funcional dentro da rotina de trabalho, por meio dos diversos recursos existentes, como áudio e imagem, que facilitam algumas atividades relacionadas ao trabalho.

Eu acredito que ela seja uma ferramenta útil pra algumas finalidades do nosso trabalho, pela eficiência que o aplicativo nos dá, velocidade da informação, não só via texto, como o áudio, que você pode utilizar. G11

Eu até brinco que a gente fica um pouco viciado no aplicativo pelo fato de você ver o que está acontecendo através das mensagens, ou até mesmo imagens, fotos, o que está acontecendo nas outras unidades, visto que náo tem como estar nelas ao mesmo tempo. G05

A Classe 5, denominada as principais potencialidades e fragilidades no uso do WhatsApp ${ }^{\oplus}$, corresponde às potencialidades e fragilidades relatadas pelos gestores no uso do aplicativo na gestão dos serviços de saúde.

As potencialidades eu acho que é a rapidez da comunicação, (...) a gente consegue fazer mais coisa em menos tempo. G02
As fragilidades é que a gente pode expor principalmente foto, e algumas situaçóes que ou ridicularizam outros, ou que se tornam fofocas, ou que expóe o próprio paciente, eu acho que isso é uma grande fragilidade. G09

A Classe 6, percepção da utilidade do aplicativo em decisóes que influenciam o processo de trabalho, expressa a insegurança no uso do aplicativo em assuntos confidenciais e/ou sigilosos, assim como em decisóes importantes e questóes que envolvam mudanças definitivas, por não ser ainda considerado o meio oficial para esse tipo de informação.

Eu acho que tomar decisóes importantes não, acho que nada substitui uma boa reunião pra discutir, olhar cara a cara as pessoas, eu acho que algumas decisóes você acaba tomando quando você precisa ser rápido, mas em coisas menores. G07

Então, eu tive uma experiência em que eu tomei uma decisão importante por WhatsApp ${ }^{\oplus}$ e teve uma repercussáo muito ruim, então a partir dessa experiência, eu optei por fazer pessoalmente, por exemplo, uma pessoa que quer uma mudança de setor, eu peço pra agendar. G06

\section{Discussão}

A mensagem instantânea possui a grande vantagem de reduzir a distância entre os integrantes e facilitar os fluxos comunicacionais, ${ }^{(14)}$ o que foi enfatizado pelos gestores em suas falas, pela possibilidade de integrar os serviços e profissionais envolvidos no processo de trabalho, tanto entre gestores e demais funcionários, como entre os trabalhadores de um mesmo setor ou área, agilizando a troca de informações e facilitando a atuação do gestor.

Em relação à escolha das tecnologias existentes, os gestores deixaram claro sua preferência pelo e-mail para utilização em assuntos formais, porém, até que o e-mail fosse considerado um canal formal para uso profissional, transcorreu um longo período. Portanto, o mesmo processo de incorporação de uma nova tecnologia pode acontecer em relação ao WhatsApp ${ }^{\oplus}$, visto sua rápida difusão no meio profissional. ${ }^{(15)}$ 
Ao serem questionados, a maioria dos gestores relataram a capacidade de se comunicar em tempo real e a possibilidade de executar várias tarefas ao mesmo tempo como principais potencialidades no uso do aplicativo. A rapidez na transmissão de informações se origina da facilidade de encaminhar uma mensagem com somente um clique na tela. ${ }^{(16)}$

Nesse sentido, estudo referente ao uso de mídias sociais em gerenciamento de projetos retrata a importância do uso dessas tecnologias, que propiciam uma comunicação constante e eficiente entre os colaboradores, e se relaciona diretamente à obtenção de resultados imediatos, afetando de forma positiva o desenvolvimento e eficiência do trabalho. ${ }^{(1)}$

Como expresso na fala dos entrevistados, ao relatarem que a partir do uso dos recursos que o aplicativo dispóe, conseguem saber em tempo real o que ocorre em outras áreas de atuação, e permite que intervenham de acordo com a necessidade sem precisar que se locomovam até o local. Entretanto, existem muitas barreiras à comunicação, como dificuldade de compreensão, excesso de informaçóes ou diferenças culturais, que podem distorcer a percepção da mensagem. ${ }^{(14)}$

O receptor, seja ele qual for, interpreta a mensagem de acordo com seu histórico de vida, experiências e desejos, projetando seus interesses e expectativas. Portanto, é compreensível que os gestores entrevistados se sintam inseguros a depender do tipo e complexidade da informação emitida. Vale ressaltar que a escolha do canal de comunicação decorre do tipo de mensagem a ser enviada, que pode ser de caráter simples e direto, ou de conteúdo complexo, com maior probabilidade de equívoco de entendimento. ${ }^{(14)}$

Nesse sentido, a comunicaçáo face a face possui a vantagem de viabilizar diversos sinais de informação como posturas, expressão facial, gestos e entonaçóes e a oportunidade do feedback imediato. ${ }^{(14)} \mathrm{O}$ que conversa com os relatos dos entrevistados, que ao tomar uma decisão importante, ou tratar de mudanças significativas no processo de trabalho, preferem se comunicar pessoalmente, para evitar falhas de comunicação.

Outro achado foi o impacto na jornada de trabalho dos gestores, visto que hoje em dia, estar conectado em um smartphone, significa estar disponível, o que permite que você seja alcançado independentemente de estar em seu horário de trabalho, inclusive aos finais de semana. Além disso, o mesmo canal de comunicação é utilizado tanto em questôes pessoais, como profissionais, transpassando a divisa entre vida pessoal e trabalho, o que afeta o tempo despendido para assuntos profissionais quando se está em casa, corroborando com resultados de outros estudos. ${ }^{(15,17,18)}$

Com essa mudança no conceito de disponibilidade do profissional, a comunicação pelo aplicativo tende ao imediatismo, o que revela uma desvantagem no uso do aplicativo encontrada em diferentes estudos, que incluem a falta de paridade do que é considerado urgente pelos sujeitos da conversa, o que pode gerar cobrança por uma resposta imediata independente do assunto ou problema. ${ }^{(16-19)}$

O grau de vulnerabilidade das informaçóes mostrou-se como fator de preocupação por parte dos gestores, em razão da possibilidade de exposição de assuntos sigilosos, fomentação de fofocas e outros ruídos que prejudicam a comunicação e podem acarretar danos à instituição e à terceiros. ${ }^{(18-20)}$ Entretanto, acrescenta-se que o WhatsApp ${ }^{\oplus}$ garante segurança e privacidade por meio da criptografia de ponta a ponta, que significa que somente os integrantes de uma conversa possuam acesso às mensagens enviadas, ${ }^{(21)}$ cabendo ao gestor a escolha pela utilização ou não do aplicativo, conforme a complexidade e grau de importância de um assunto.

Além disso, os serviços de saúde devem estar preparados, no sentido de discutir sobre o uso adequado de mídias sociais no ambiente profissional, fornecendo orientaçóes e abordando as consequências do mal uso, como a violação de políticas, que incluem vazamento de informaçóes confidenciais, seja de pacientes ou da própria instituição. ${ }^{(18-20)}$

Como limitaçóes, não foram encontrados estudos que abordassem as implicaçóes práticas para gestores de serviços de saúde, decorrentes do uso de redes sociais como o WhatsApp ${ }^{\oplus}$ em seu processo de trabalho.

Considerando as vantagens no uso do WhatsApp ${ }^{\oplus}$, observou-se que esse aplicativo se destaca enquanto uma ferramenta essencial, complementar ao uso de e-mails. No entanto, existe a necessidade de se conhecer as implicaçóes trabalhistas para a sua formalização enquanto ferramenta de uso legal de comunicação. 


\section{Conclusão}

O estudo descreveu como os gestores de serviços de saúde utilizam o WhatsApp ${ }^{\oplus}$ na sua atuação profissional, evidenciando a importância do aplicativo enquanto ferramenta estratégica na gestão dos serviços de saúde, a partir do compartilhamento e integração de informaçóes em tempo real, assim como a aproximação dos colaboradores e serviços envolvidos no processo de trabalho, facilitando a atuação do gestor por meio dos diversos recursos existentes no aplicativo. Embora as inúmeras potencialidades percebidas pelos entrevistados, algumas desvantagens foram pontuadas, como a tendência ao imediatismo e a cobrança por disponibilidade 24 horas, sendo importante a imposição de limites para preservar o equilíbrio entre vida pessoal e profissional, além do uso adequado do aplicativo, evitando possíveis rumores no ambiente de trabalho.

\section{Agradecimentos}

Ao Conselho Nacional de Ciência e Tecnologia $(\mathrm{CNPq})$, pelo apoio financeiro através do Programa Institucional de Bolsas de Iniciação Científica (PIBIC), que possibilitou a realização desse estudo.

\section{Colaborações}

Savio RO, Barreto MFC, Pedro DRC, Costa RG, Rossaneis MA, Silva LGC, Aroni P e Haddad MCFL colaboraram com a concepção do estudo, análise e interpretação dos dados, redação do artigo, revisão crítica relevante do conteúdo intelectual e aprovação da versão final a ser publicada.

\section{Referências}

1. Hysa B, Spalek S. Opportunities and threats presented by social media in project management. Heliyon. 2019;5(4):e01488.

2. Mesquita AC, Zamarioli CM, Fulquini FL, Carvalho EC, Angerami EL. Social networks in nursing work processes: an integrative literature review. Rev Esc Enferm USP. 201;51:e03219.
3. Lima IC, Galvão MT, Pedrosa SC, Cunha GH, Costa AK. Uso do aplicativo WhatsApp no acompanhamento em saúde de pessoas com HIV: uma análise temática. Esc Anna Nery. 2018;22(3):e20170429.

4. Macêdo DF, Romeiro TI, Marsiglia DC. A importância do administrador na gestão hospitalar: Percepção de médicos, enfermeiros e administradores de um hospital universitário. Rev F0C0. 2015;8(2):37.

5. Senhoras EM. A cultura na organização hospitalar e as políticas culturais de coordenação de comunicação e aprendizagem. Rev Eletr Comun Inform Inov Saúde. 2017;1(1):45-55.

6. WhatsApp [Internet]. Sobre o WhatsApp. [citado 2020 Dez 5]. Disponível em: https://www.whatsapp.com/about/

7. Pinto LF, Rocha CM. Inovações na Atenção Primária em Saúde: o uso de ferramentas de tecnologia de comunicação e informação para apoio à gestão local. Ciênc Saúde Colet. 2016;21(5):1433-48.

8. Paulino DB, Martins CC, Raimondi GA, Hattori WT. WhatsApp $®$ como recurso para a educação em saúde: contextualizando teoria e prática em um novo cenário de ensino-aprendizagem. Rev Bras Educ Med. 2018;42(1):171-80.

9. Kakushi LE, Evora YD. As redes sociais na educação em enfermagem: revisão integrativa da literatura. Rev Lat Am Enfermagem. 2016;24e2709.

10. Chan WS, Leung AY. Use of social network sites for communication among health professionals: systematic review. J Med InternetRes. 2018;20(3):e117.

11. Tong A, Sainsbury P, Craig J. Consolidated criteria for reporting qualitative research (COREQ): a 32-item checklist for interviews and focus groups. Int J Qual Health Care. 2007;19(6):349-57.

12. Ratinaud P. IRAMUTEQ: Interface de $R$ pour les analyses multidimensionnelles de textes et de questionnaires. 2018. [citado 2020 Nov 20]. Disponível em: http://www.iramuteq.org

13. Camargo BV, Justo AM. Tutorial para uso do software de análise textual IRAMUTEQ. Florianópolis: Universidade Federal de Santa Catarina; 2018. [citado 2020 Nov 20]. Disponível em: http://www.iramuteq.org/ documentation/fichiers/tutoriel-en-portugais

14. Robbins SP. Comunicação. In: Robbins SP, editor. Comportamento organizacional. 11a ed. São Paulo: Pearson Education do Brasil; 2009. p. 231-55.

15. Carvalho AP. Midiatização nas relações de trabalho e as práticas de comunicação de grupos organizacionais em dispositivos móveis: 0 caso WhatsApp [dissertação]. Curitiba: Universidade Tuiuti do Paraná; 2017.

16. Khanna V, Sambandam SN, Gul A, Mounasamy V. "WhatsApp"ening in orthopedic care: a concise report from a 300-bedded tertiary care teaching center. Eur J Orthop Surg Traumatol. 2015;25(5):821-6.

17. Maciel MA, Bobsin D, Behr A. A influência da comunicação eletrônica na vida pessoal e profissional do servidor público da Universidade Federal Do Pampa - Campus Sant'ana Do Livramento. VII Congresso Virtual Brasileiro - Administração. 2012 [citado 2019 Ago 20]. Disponível em: http://www.convibra.com.br/artigo.asp?ev=22\&id=4529

18. Mars M, Scott RE. WhatsApp in clinical practice: a literature review. Stud Health Technol Inform. 2016;231:82-90.

19. De Benedictis A, Lettieri E, Masella C, Gastaldi L, Macchini G, Santu C, et al. WhatsApp in hospital? An empirical investigation of individual and organizational determinants to use. PLoS One. 2019;14(1):e0209873

20. Ganasegeran K, Renganathan P, Rashid A, Al-Dubai SA. The m-Health revolution: exploring perceived benefits of WhatsApp use in clinical practice. Int J Med Inform. 2017;97:145-51.

21. WhatsApp [Internet]. 2020. WhatsApp Encryption Overview: Technical white paper. [citado 2020 Dez 5]. Disponível em https://www. whatsapp.com/security/WhatsApp-Security-Whitepaper.pdf 